\title{
Syed Mohibur Rab
}

Unfortunately the iPad version of this Obituary ( $B M J$

2014;349:g5999, doi:10.1136/bmj.g5999) showed the wrong picture when it was first published. We have now corrected this error, but if you are reading The $B M J$ on an iPad you may have to refresh your weekly issue in order to see the corrected version.
Cite this as: BMJ 2014;349:96765

๑ BMJ Publishing Group Ltd 2014 